\title{
Long-term follow-up of penile curvature correction utilizing autologous albugineal crural graft
}

\author{
Carlos Teodósio Da Ros,Túlio Meyer Graziottin, Eduardo Ribeiro, Márcio Augusto Averbeck
}

Centro de Andrologia e Urologia, Porto Alegre, Brazil

\section{ABSTRACT}

Purpose: Peyronie's disease is an acquired connective tissue disorder of the penile tunica albuginea with fibrosis and inflammation. The disease produces palpable plaques, penile curvature and pain during erections. Usually it results in impairment of the quality of life. Our objective is to review the long-term results of the albugineal grafting harvested from the penile crura for the treatment of severe penile curvature.

Materials and Methods: Thirty-three patients with Peyronie's disease were submitted to a grafting with tunica albuginea from the penile crura for the correction of penile curvature. The results were evaluated after 6 months of the procedure. Variables studied were overall satisfaction with the procedure, correction of the penile curvature, erectile capacity, penile shortening and the presence of surgical complications.

Results: Mean follow-up after surgery was 41 months. Complete correction of the curvature was achieved in 30 patients (90\%). The mean preoperative curvature was 91.8 degrees and median plaque length was $2 \mathrm{~cm}$ (ranged from 1 to $5 \mathrm{~cm}$ ). Three patients (9\%) experienced recurrence of the penile curvature and required a new procedure. In 30 men (90\%) the procedure fulfilled their expectations and in 31 patients (93.9\%) their opinions were that sexual partners were satisfied with the penile correction. Penile shortening or augmentation was referred in 6 $(18.1 \%)$ and $1(3 \%)$ patient, respectively.

Conclusion: Our series demonstrated that grafting the albugineal defect after incision of the tunica albuginea with tunica from the crus for the correction of penile curvature is safe and results in satisfactory straight erections duringa long-term follow-up.

\section{ARTICLE INFO}

\section{Key words:}

Peyronie Disease; Tunica

albuginea; Penile Induration

Int Braz J Urol. 2012; 38: 242-9

Submitted for publication:

April 11, 2011

Accepted after revision:

August 12, 2011

\section{INTRODUCTION}

Penile curvature is the result of a congenital penile defect in tunica albuginea, trauma, or Peyronie's disease (PD). While congenital curvatures affect patients in their early sexual life, PD is more frequent after the fortieth decade. PD is an acquired connective tissue disorder of the tunica albuginea, and the pathophysiology is not perfectly understood $(1,2)$. The development of fibrosis and inflammation of the tunica albuginea produces palpable plaques, penile curvature and pain during erections. Sexual dysfunction due to erectile impairment or penile curvature can accompany PD in some cases, and it results in negative impact of the quality of life in two thirds of the patients (3). In the presence of severe curvatures, the deformity of the penis interferes with penile 
penetration, resulting in difficult coitus. Patients with this condition frequently report shame, embarrassment and interpersonal difficulties (4).

The etiology of PD is probably multifactorial. Repetitive and undetected microtraumas during coitus result in delamination between the layers of the tunica albuginea, microvascular injury, hemorrhage, and local activation of inflammatory and fibrotic pathways (5). PD is a relatively common situation that can affect almost $10 \%$ of the adult male population (6). PD is initially characterized by an inflammatory phase associated with painful erections, bending, or a change in plaque size. During this phase (6-18 months), the condition may progress, stabilize, or regress. As long as the inflammation in the plaque subsides, pain tends to disappear (7).

In patients with a slight curvature and no erectile dysfunction (ED), treatment is conservative (1). Medical treatment should be utilized in the inflammatory phase of the disease, although weak evidence of improvement is reported in the literature (2).

Surgical correction of PD is reserved for patients with curvature or narrowing of the tunica albuginea, which interferes with sexual intercourse after plaque stabilization. At least three months of stabilization is required. Currently surgery includes shortening of the convex tunica (shortening procedures), or incision of the plaque with subsequent grafting (lengthening procedures). In a case with PD and severe ED, insertion of a penile prosthesis is preferred. Lengthening procedures are more complex than shortening procedures, and preferentially utilized in patients with severe penile curvature, hourglass deformities, or unacceptable penile shortening $(8,9)$. After the plaque incision for lengthening of the concave side, a graft should be sutured in the defect. Several grafting materials have been used like dermis, fascia temporalis, dura mater, tunica vaginalis, saphenous vein, bovine and human cadaveric pericardium, porcine small intestine submucosa, and synthetic materials (polyester and polytetrafluoroethylene) (10). The perfect substitute to fill the tunica defect is still under research; nevertheless we believe that the healthy tunica can be the ideal graft, as previously described (11).
The aim of the present study is to review our long-term results of the surgical grafting with tunica albuginea retrieved from the penile crura for the treatment of symptomatic penile curvature.

\section{MATERIALS AND METHODS}

Between June 2002 and October 2009, 33 patients with Peyronie's disease were submitted to correction of the penile curvature with grafting of crural tunica albuginea as previously described (11). Surgical candidates were patients with stable Peyronie's disease for at least one year and unable to achieve sexual penetration due to curvature, and those in which medical management had failed, as stated previously (12). Patients had curvatures greater than 75 degrees determined with preoperative photography of erections (Figure-1), and all were candidates to a lengthening procedure. Medical treatment utilized before surgery included oral colchicine, vitamin E or tamoxifen, or intralesional verapamil. Patients should have good penile rigidity, with or without phosphodiesterase inhibitors.

Briefly, the patient was placed in the lithotomy position, penile skin was incised (circumcision) and the penis was completely degloved. A proximal tourniquet was applied on the penis, and

\section{Figure 1 - Measurement of the angle based on patient's picture.}

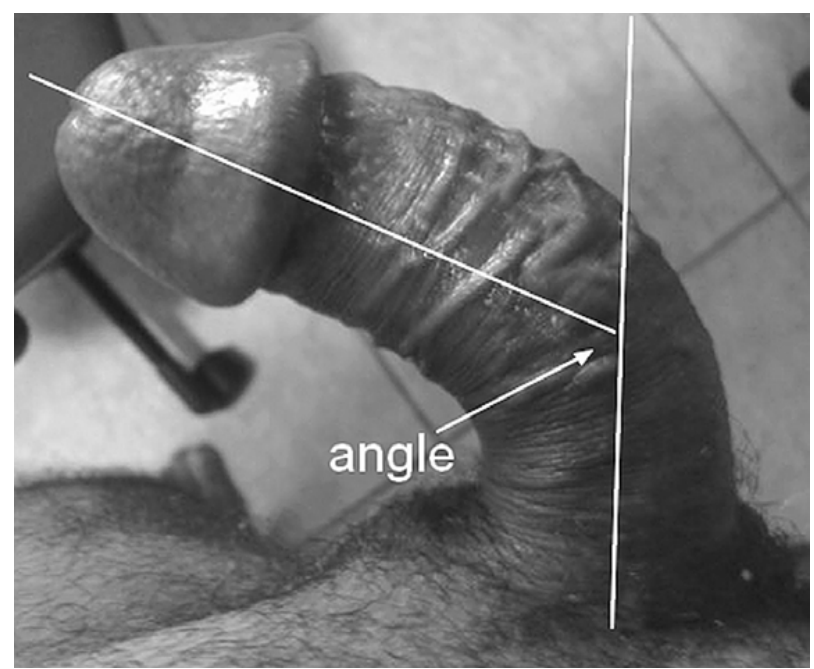


saline solution was infused through a 19-gauge needle in the corpus cavernosum to produce an artificial erection (Figure-2). The dorsal neurovascular bundle and urethra, when necessary, were dissected free from the tunica albuginea (Figure-3). The exposed fibrous plaque and occasionally the septum of corpora cavernosa were incised under traction of the glans with the objective of obtaining the maximum length. At the edges of the gap, the smooth muscle was dissected from the tunica albuginea. A $3 \mathrm{~cm}$ perineal incision was made, one or both crura were identified, and a segment of the crural albuginea was dissected from the corpus cavernosum (Fig-

Figure 2 - Induced erection to observe the curvature.

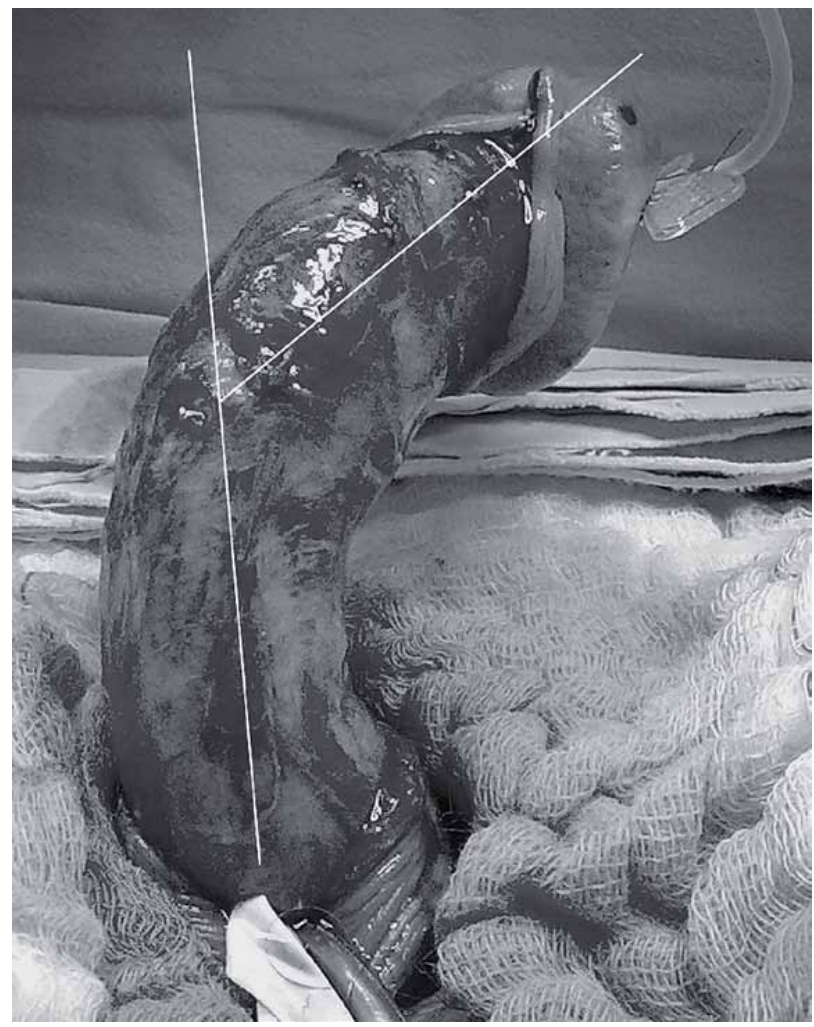

ure-4). Bilateral crural graft was utilized if necessary. The donor area was watertight sutured with polyglactin 2-0 (Vicryl $\left.{ }^{\circledR}\right)$. The graft was prepared removing all residual ischiocavernous muscle. Grafting in the penis was performed with running sutures with polyglactin 4-0 (Vicryl $\left.{ }^{\circledR}\right)$ (Figure-5). Artificial erection was performed to assure cur- vature correction, and complementary plication of the tunica was utilized if necessary (Figure-6). Coitus was delayed for at least 30 days. All patients were operated by one surgeon (CTDR).

Patients were interviewed on 3 and 6 months and results were evaluated after 6 months from the procedure. Variables studied were overall satisfaction with the procedure, penile straightness, erectile capacity, penile shortening and the presence of complications. Data were presented as descriptive statistics.

The study was approved by the local IRB and written informed consent was obtained from all subjects.

Figure 3 - It is necessary to detach neurovascular bundle or urethra to permit the access to the Peyronie's plaque.

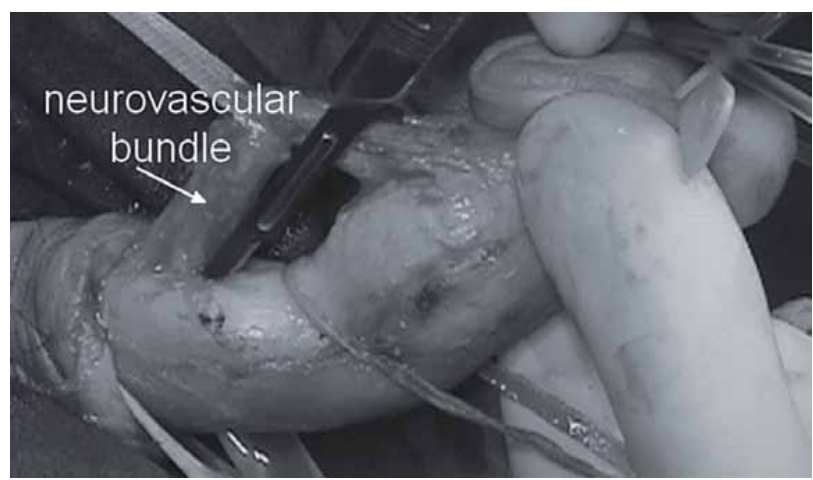

Figure 4 - After the perineal incision, we can see the urethra and penile crus.

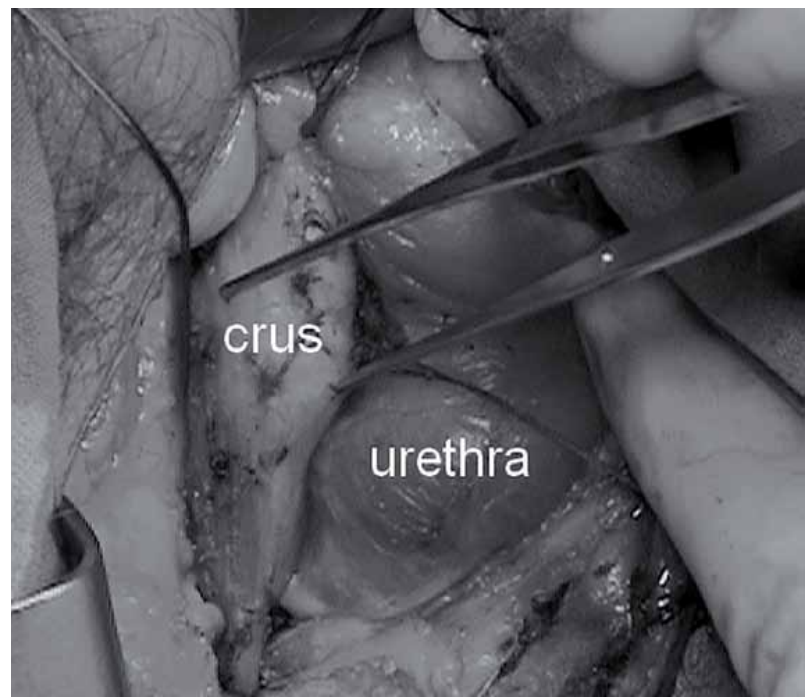


Figure 5 - The tunical graft sutured over the penile defect.

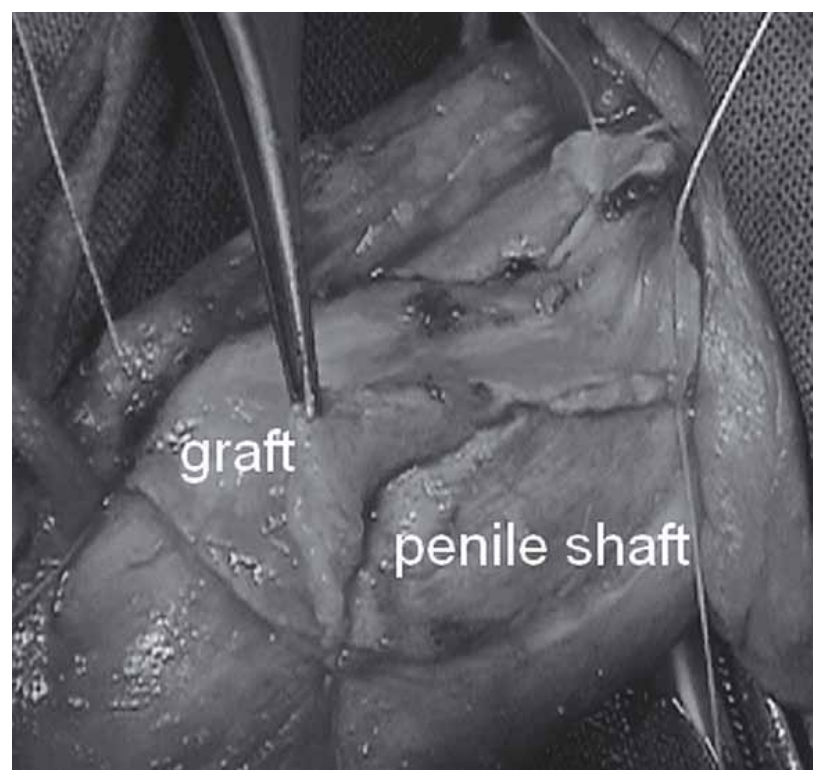

\section{RESULTS}

The mean and standard deviation age were $54.2 \pm 15.1$ years. Mean follow-up time after surgery was 41 months, ranging from 9 to 96 months. Complete correction of the curvature was achieved in 30 patients (90\%). The mean and standard deviation of preoperative curvature were 91.8 and 18.7 degrees (ranged 75 to 160 degrees). Three patients (9\%) experienced recurrence of the penile curvature and required a new procedure (plicature of the tunica) and the final result was a straight penis. The mean and standard deviation of postoperative curvature was 4.5 and 6.2 degrees. The median size of the plaque was $2 \mathrm{~cm}$ (ranged from 1 to $5 \mathrm{~cm}$ ).

Table-1 summarizes the results. Thirty patients (90\%) answered that they would do the procedure again. Also, in 30 men (90\%) the procedure fulfilled their expectations and in 31 patients $(93.9 \%)$ their opinions were that sexual partners were satisfied with the penile correction.

Six patients (18.1\%) complained of impairment of erectile function and received phosphodiesterase 5 inhibitors or intracavernosal therapy. Two patients (6.06\%) developed refractory ED. Penile shortening or augmentation was informed by $6(18.1 \%)$ and $1(3 \%)$ patients respectively, while
Figure 6 - Artificial erection to check the procedure.

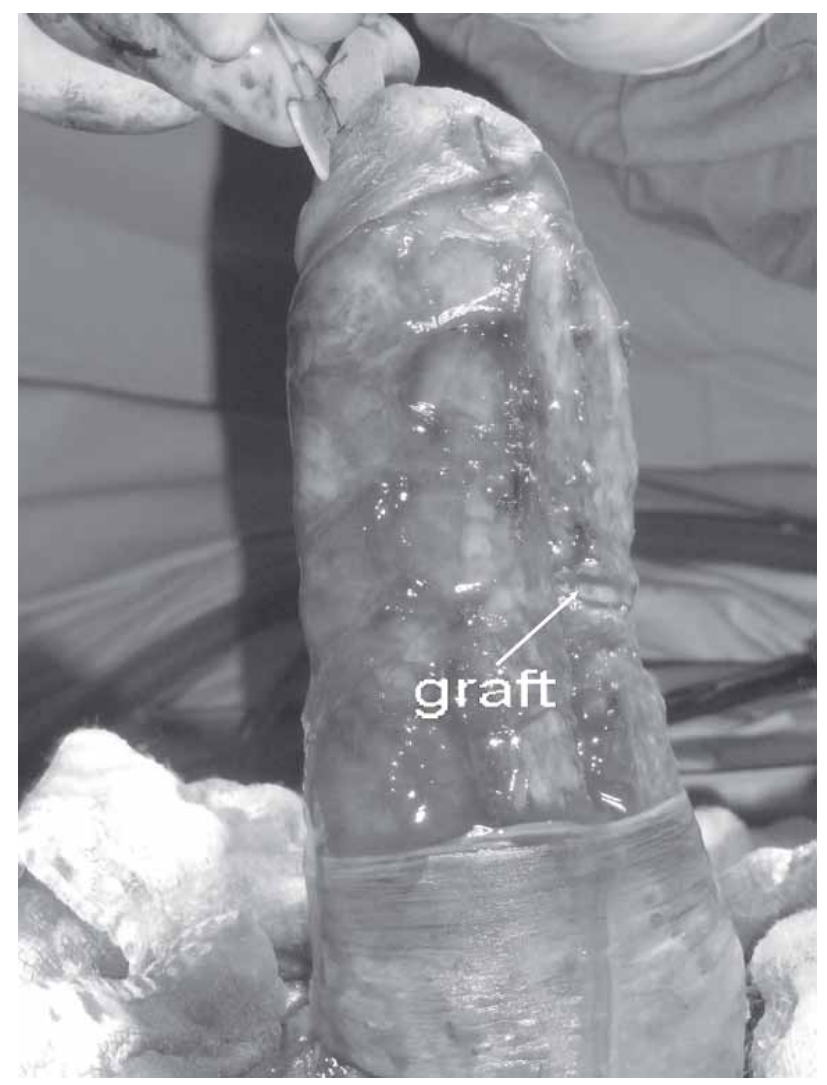

26 patients $(78.8 \%)$ stated no difference in penile size. Six patients (18.1\%) complained of hypoesthesia on glans and $4(12.1 \%)$ had penile pain or discomfort on intercourse. No patient had wound infection, urethral injury or penile nodules.

The main reason for dissatisfaction with the procedure was recurrence of the curvature (1 patient), impairment of erectile function (1 patient), and glans hypoesthesia (1 patient).

\section{DISCUSSION}

Peyronie's disease has an important impact over the quality of life of the patients. Patients report negative effects in four major domains: physical appearance and self-image, sexual function and performance, pain and social stigmatization (4).

Surgical correction of the curvature is based on lengthening the penis, incision or excision of the plaque with grafting in the concave side, or shortening of the penis, using plication 
Table 1 - Pre and post operative curvatures.

\begin{tabular}{lcc}
\hline Curvatures (degrees) & Pre operative $\mathrm{n}$ & Post operative $\mathrm{n}$ \\
\hline $75-90$ & 26 & - \\
$91-120$ & 6 & - \\
$120-160$ & 1 & - \\
$0-10$ & - & 31 \\
$11-20$ & - & 2 \\
\hline
\end{tabular}

sutures in the convex side of the curvature. Tunical shortening procedures, such as Nesbit corporoplasty, present low morbidity and are faster surgeries than grafting; however they result in significant loss of penile length (13). We prefer lengthening procedures in situations where penile shortening is expected with Nesbit plicature, like curvatures with angle greater than 45 degrees or hourglass defect.

Penile shortening was subjectively informed by $18.1 \%$ of the patients. We explained carefully, before the surgery, that the objective of the procedure was to straight the penis or correct the hourglass defect. In the medical literature, there is no consensus on how to measure the penile length in a curved penis with Peyronie's disease. We can stretch the flaccid penis or measure it in full erection injecting saline. The majority of the authors measure it from the pubis to the end of the glans penis. Some problems are envisioned with these length measurements. First, pubic fat can interfere in the measurement. Second, the strength utilized to pull the flaccid penis can interfere in obtain reliable data. Third, when we measure a full erected curved penis, we would obtain a section of a perimeter of a circle (in the convex side), since the measurement in straight line is not a true measure of penile length. Fourth, the surgery is not developed to increase the penile length, and we explain that to the patients and that the main objective is to obtain a straight penis. Grafting the concave side will allow us to obtain an erection with the length of the convex side.

This study describes the long-term results of grafting with tunica albuginea, the grafting technique of choice in our service. After more than 3 years of follow-up, we observed that 90\% of our patients maintained a straight penis and were satisfied with the procedure.

The investigation for the ideal graft for penile reconstruction has demonstrated that the best tissue to substitute the sick albuginea is the patient albuginea $(11,13)$. The albuginea of the penile crura can be retrieved in different sizes and from both sides, is ready to use, is autologous, and has no extra cost. Moreover, we think that normal function of the tunica albuginea like tensile strength and elasticity needs an architecture of collagen and elastic fibers, and crural graft fulfills these requirements. As a matter of fact, none of our patients developed penile bulging or narrowing.

The crural grafting technique avoids costs allografts or xenografts. In addition, any urologist familiarized with the perineal anatomy should be able to harvest the tissue, and no major morbidity is expected with this approach. Pain in the donor site is limited to the first days of surgery, and perineal scaring has minimal cosmetic effect.

Hypoesthesia of the glans had similar rates of other lengthening techniques, and it is probably related to the neurovascular bundle dissection. El-Sakka et al. reported a 10\% rate of diminished glans sensation among patients that underwent a plaque incision and venous grafting (14), while Taylor and Levine (15) found 31\% of diminished penile sensation with plication and pericardial grafting. In our series 18\% of the patients complained of glans hypoesthesia.

Peyronie's disease is a risk factor for erectile dysfunction. However, there are two poten- 
tial causes to increase erectile dysfunction with our technique. First, the perineal dissection of the crura could damage the penile neurovascular bundles. We avoid dissecting close to the ischium bone, keeping the harvesting of the tunica close to the ischiocavernous muscle. Second, the diminushion of the crural compartment of the corpus cavernosum can impair the haemodynamics of the penis. We did not find a higher rate of erectile dysfunction in our series, even when both crural tunicas are harvested. We believe that crural portion of corpora cavernosa can be reduced without any harm. Even a penile prosthesis can be implanted after the procedure with a reduction of the prosthesis rod. Also, we could not find any case of fibrotic plaques on the crura, and all patients had healthy albuginea.

\section{CONCLUSIONS}

Our series demonstrated that grafting the albugineal defect after incision of the tunica albuginea in lengthening surgeries of penile curvatures with crural tunica albuginea is safe and results in satisfactory straight erections in a longterm follow-up.

\section{CONFLICT OF INTEREST}

None declared.

\section{REFERENCES}

1. Gholami SS, Gonzalez-Cadavid NF, Lin CS, Rajfer J, Lue TF: Peyronie's disease: a review. J Urol. 2003; 169: 1234-41.

2. Vardi $Y$, Levine LA, Chen J, Hatzimouratidis K, Sohn M: Is there a place for conservative treatment in Peyronie's disease? J Sex Med. 2009; 6: 903-9.

3. Gelbard MK, Dorey F, James K: The natural history of Peyronie's disease. J Urol. 1990; 144: 1376-9.

4. Rosen R, Catania J, Lue T, Althof S, Henne J, Hellstrom W et al.: Impact of Peyronie's disease on sexual and psychosocial functioning: qualitative findings in patients and controls. J Sex Med. 2008; 5: 1977-84.

5. Bekos A, Arvaniti M, Hatzimouratidis K, Moysidis K, Tzortzis V, Hatzichristou D: The natural history of Peyronie's disease: an ultrasonography-based study. Eur Urol. 2008; 53: 644-50.

6. Smith CJ, McMahon C, Shabsigh R: Peyronie's disease: the epidemiology, aetiology and clinical evaluation of deformity. BJU Int. 2005; 95: 729-32.

7. Bivalacqua TJ, Purohit SK, Hellstrom WJ: Peyronie's disease: advances in basic science and pathophysiology. Curr Urol Rep. 2000; 1: 297-301.

8. Pryor J, Akkus E, Alter G, Jordan G, Lebret T, Levine L et al.: Peyronie's disease. J Sex Med. 2004; 1: 110-5.

9. Mulhall J, Anderson M, Parker M: A surgical algorithm for men with combined Peyronie's disease and erectile dysfunction: functional and satisfaction outcomes. J Sex Med. 2005; 2 : 132-8.
10. Kadioglu A, Sanli O, Akman T, Ersay A, Guven S, Mammadov F: Graft materials in Peyronie's disease surgery: a comprehensive review. J Sex Med. 2007; 4: 581-95.

11. Teloken C, Grazziotin T, Rhoden E, Da Ros C, Fornari A, Soares FC et al.: Penile straightening with crural graft of the corpus cavernosum. J Urol. 2000; 164: 107-8.

12. Lue TF, Giuliano F, Montorsi F, Rosen RC, Andersson KE, Althof $S$ et al.: Summary of the recommendations on sexual dysfunctions in men. J Sex Med. 2004; 1: 6-23.

13. Kendirci M, Hellstrom WJ: Critical analysis of surgery for Peyronie's disease. Curr Opin Urol. 2004; 14: 381-8.

14. El-Sakka AI, Rashwan HM, Lue TF: Venous patch graft for Peyronie's disease. Part II: outcome analysis. J Urol. 1998; 160: 2050-3. Erratum in: J Urol 1999; 162: 809.

15. Taylor FL, Levine LA: Surgical correction of Peyronie's disease via tunica albuginea plication or partial plaque excision with pericardial graft: long-term follow up. J Sex Med. 2008; 5: 2221-8; discussion 2229-30.

Correspondence address:

Dr. Carlos Teodósio Da Ros

Ferdinand Kisslinger, 80 / 302B

Porto Alegre. 91360-054, RS, Brazil

FAX: +55 51 3378-9996

E-mail carlos.da.ros@terra.com.br 


\section{EDITORIAL COMMENTS}

Peyronie's disease is characterized by different penile deformities caused by albugineal fibrosis and shrinkage. The disease is often associated with penile shortening. It has major influence on quality of life and is often associated with serious psychological consequences. The incidence is $3-10 \%$ among the male population; it can be commonly found in men between 40 to 70 years; however it can also affect young population under 30 years (1). According to Usta et al., Peyronie's disease is commonly associated with erectile dysfunction, ranging from 20 to 54\% (2).

The surgery is indicated only to stable cases, which is not been clearly defined, but is generally accepted to be at least one year of disease and at least six months where there has been no change in the deformity and that pain has resolved. The surgical candidate should also describe a compromise or inability to engage in coitus secondary to deformity or inadequate rigidity. In addition, a patient who has medical treatment failure and has extensive plaque calcification is a proper candidate for surgery. Surgical treatment must be individualized, aiming not only at restoring penile function, but also restoring as much as possible the previous shape of the penis. Assessment of the erectile capacity is essential to decide the type of operation. Furthermore, patients' expectation should be considered and informed consent should be obtained.

Different surgical techniques have been proposed for penile curvature correction. In general they are divided in Tunical-shortening procedures (Nesbit and its modifications, dot-plication and Yachia procedures), Tunical-lengthening procedures which include excision, incision and grafting; and insertion of penile prosthesis grafting. Lengthening procedures are more complex than shortening procedures, and preferentially utilized in patients with severe penile curvature, hourglass deformities, or unacceptable penile shortening.

Different grafts materials were suggested to cover the tunical defect, and can be classified into 2 classes:
- Autologous graft:(dermal, tunica vaginalis flap, fascia lata, muscularis aponeurosis, bucal mucosa and veins, including deep dorsal vein, saphenous vein and tunica albuginea graft.

- Allograft tissue and synthetic materials: cadaveric or bovine pericardium, Dacron and Gore-Tex are the most reported.

At present time, none of them are the gold standard treatment. The ideal graft should be readily available, easily sutured, pliable, compliant, inexpensive, well tolerated and with minimal tissue reaction.

In general when Tunica albuginea is used, excision should be made in the corpora. One should have in mind that this could cause narrowing of the proximal corpus cavernosum. There for this procedure should only be used for small corporal defects. According to Teloken et al the defect should not be larger than $3.5 \times 2.0 \mathrm{~cm}$ (3). In this study the median plaque size was 2 $\mathrm{cm}$. Furthermore, this procedure may weaken the support of the penis and increase the complexity of future penile prosthesis implantation (3).

In this study the authors describe long term results of operative therapy for Peyronie's disease of 33 patients by using graft of the tunica albuginea retrieved from the crura of the penis. This method has been already described before, but there are only few long term results available in the literature.

All patients had severe curvature which unable them to have sexual intercourse. After the operation $90 \%$ of the patients were satisfied with the operation, which is comparable with previous published results of this operation technique, with shorter follow-up (4-6). Still 18\% complained of hypoesthesia of the glans which is known from the literature to be a common complication. Unfortunately there was no report about penile length before and after the operation.

In this study the authors demonstrated that this operation method is safe and achieved satisfactory results in the majority of the patients and therefore I want to congratulate them. 


\section{REFERENCES}

1. Smith CJ, McMahon C, Shabsigh R: Peyronie's disease: the epidemiology, aetiology and clinical evaluation of deformity. BJU Int. 2005; 95: 729-32.

2. Usta MF, Bivalacqua TJ, Tokatli Z, Rivera F, Gulkesen KH, Sikka SC, et al.: Stratification of penile vascular pathologies in patients with Peyronie's disease and in men with erectile dysfunction according to age: a comparative study. J Urol. 2004; 172: 259-62.

3. Kadioglu A, Sanli 0, Akman T, Ersay A, Guven S, Mammadov F: Graft materials in Peyronie's disease surgery: a comprehensive review. J Sex Med. 2007; 4: 581-95.
4. Teloken C, Grazziotin T, Rhoden E, Da Ros C, Fornari A, Soares FC, et al.: Penile straightening with crural graft of the corpus cavernosum. J Urol. 2000; 164: 107-8.

5. Schwarzer JU: The tunica-albuginea-patch-technique: a new technique of an autologous grafting procedure for patients with peyronie's disease. J Urol. 2005; 173: 202, A: V742.

6. Da Ros C, Graziottin M, Ribeiro E, Bonfanti A, Sogari P, Teloken C: Graft of crural tunica albuginea for the treatment of Peyronie's disease. J Urol. 2005; 173: 202, A: V743.

Dr. Y. Reisman Men's Health Clinic, Slotervaart Hospital Louwesweg 6 1066EC, Amsterdam The Netherlands Fax + $3120512-5196$ E-mail: c.reisman@planet.nl 